\title{
Recombination deficient mutant of Caulobacter crescentus
}

\author{
Edward A. O'Neill*, Robert H. Hynes, and Robert A. Bender \\ Department of Cellular and Molecular Biology, Division of Biological Sciences, The University of Michigan, \\ Ann Arbor, MI 48109, USA
}

\begin{abstract}
Summary. A recombination-deficient $\left(\mathrm{Rec}^{-}\right)$strain of Caulobacter crescentus has been isolated from a collection of mutants sensitive to ultraviolet irradiation. The $\operatorname{Rec}^{-}$mutant fails to give recombinants following $\emptyset \mathrm{Cr} 30$-mediated generalized transduction or following RP4-mediated conjugation. The recombination frequency in the $\mathrm{Rec}^{-}$strain is at least 5000-fold lower than in the wild type strains. The $\mathrm{Rec}^{-}$mutant is indistinguishable from wild type in terms of morphology, growth rate, viability, and phage sensitivities, differing only in properties known to be associated with rec $A$-type mutations in other organisms: recombination frequency, ultraviolet sensitivity, and Weigle reactivation. The map location of the rec-526 allele has not been identified, but rec-526 can be cotransferred with the fla-169 mutation by RP4-mediated conjugation at low frequency. This apparent linkage has been used to move the rec mutation to other strains. The $\mathrm{Rec}^{-}$mutant resembles $\mathrm{rec} A$ strains of other organisms and provides a healthy strain severely deficient in recombination for use in complementation and cloning studies involving $C$. crescentus.
\end{abstract}

\section{Introduction}

The Gram-negative bacterium Caulobacter crescentus is regarded as a useful model system for studying cellular differentiation (Poindexter 1964; Bender et al. 1980). The Caulobacter cell cycle consists of a program of spatially and temporally regulated events terminating in asymmetric cell division. Caulobacter is unusual in that these events are intrinsic to the cell cycle and occur throughout exponential growth. The molecular nature of the events that regulate the developmental program is currently being investigated using genetic analysis (Barrett et al. 1982) as well as molecular cloning (Milhausen et al. 1982; Ohta et al. 1984; Purucker et al. 1982). In these analyses, genetic functions are identified by the ability to complement well-characterized mutations. Both of these approaches have been limited by the lack of a $C$. crescentus strain deficient in homologous recombination, since it is time-consuming to distinguish complementation from recombination in $\mathrm{Rec}^{+}$cells.

\footnotetext{
* Present address: Department of Molecular Biology and Genetics, The Johns Hopkins University School of Medicine, Baltimore, MD 21205, USA

Offprint requests to: R.A. Bender
}

Mutations in the recA gene of Escherichia coli virtually abolish homologous recombination and simultaneously cause sensitivity to ultraviolet (UV) irradiation (Little and Mount 1982). Many different bacterial species have genes analogous to the recA gene of E. coli (Better and Helinski 1983). We assumed that the analogy might extend to $C$. crescentus as well and that among a collection of UV-sensitive $\left(\mathrm{UV}^{\mathrm{s}}\right)$ mutants we might find a recombination deficient strain. We report here the isolation and characterization of one such UV strain of C. crescentus in which homologous recombination is reduced more than 5000 -fold.

Isolation and characterization of strain CM5256. The wildtype strain CM5000 was mutagenized by irradiation with UV light ( $\left.30 \mathrm{~J} / \mathrm{m}^{2}\right)$, resulting in a $10^{-3}$ survival. The irradiated cells were resuspended in PYE broth (Poindexter 1964), incubated overnight at $30^{\circ} \mathrm{C}$, diluted, spread on PYE agar plates, and incubated at $30^{\circ} \mathrm{C}$ until small colonies appeared. Each plate was replicated to two fresh PYE plates, one of which was irradiated with $10 \mathrm{~J} / \mathrm{m}^{2}$ of UV light from a germicidal lamp. Both replicate plates were then incubated at $30^{\circ} \mathrm{C}$ and examined periodically for colonies that grew on the unirradiated plate but not on the irradiated one. UV sensitive mutants occurred at a frequency of about $10^{-4}$.

Strain CM5256 is one of the $\mathrm{UV}^{\mathrm{s}}$ strains detected by this method. Strain CM5256 is considerably more sensitive to UV irradiation than its wild type parent strain (Figure 1). The generation time and the ratio of viable cells to turbidity are indistinguishable between strain CM5256 and CM5000 (data not shown). Strain CM5256 is as sensitive to a variety of Caulobacter phages as the wild type strain CM5000, since $\emptyset \mathrm{Cbk}, \emptyset \mathrm{Cb} 5, \emptyset \mathrm{Cr} 40$, and the generalized transducing phage øCr30 have the same efficiency of plating on both strains (data not shown). Strain CM5256 remains prototrophic and is morphologically indistinguishable from wild type in either phase contrast or electron microscopy (data not shown).

Strain CM5256 is defective in recombination: $\varnothing \mathrm{Cr} 30 \mathrm{me}$ diated transduction. The recombination efficiency of strain CM5256 was tested by its ability to be transduced to Kanamycin-resistance $\left(\mathrm{Km}^{r}\right)$ by a lysate of $\phi \mathrm{Cr} 30$ grown on strain SC1028 (fla-188::Tn5, str-152) see Table 1. The fla188::Tn5 allele simultaneously renders SC1028 non-motile and $\mathrm{Km}^{\mathrm{r}}$. In addition, this particular fla:: $\mathrm{Tn} 5$ allele results in noticeable filamentation (unpublished observation). 
Table 1. Strain list

\begin{tabular}{lll}
\hline Strain & Genotype & Source or comments \\
\hline C. crescentus & & \\
CM5000 & $+(=\mathrm{CB} 15)^{\mathrm{a}}$ & B. Ely (Poindexter 1964) \\
CM5210 & uvr-501 & UV mutagenesis of CM5000 \\
CM5219 & uvr-502 & UV mutagenesis of CM5000 \\
CM5234 & uvr-504 & UV mutagenesis of CM5000 \\
CM5246 & uvr-516 & UV mutagenesis of CM5000 \\
CM5248 & uvr-518 & UV mutagenesis of CM5000 \\
CM5252 & uvr-522 & UV mutagenesis of CM5000 \\
CM5256 & rec-526 & UV mutagenesis of CM5000 \\
CM5281 & rec-526 fla-169::Tn5 & SC1759 $\rightarrow$ CM5256 \\
& (pVS1) & \\
CM5282 & str-501 & Spont. Sm ${ }^{\mathrm{R}}$ of CM5000 \\
CM5283 & fla-169::Tn5 (pVS1) & SC1759 $\rightarrow$ CM5000 \\
CM5284 & proC104 str-502 & Spont. Sm ${ }^{\mathrm{R}}$ of SC451 \\
CM5285 & rec-526 fla-169::Tn5 & CM5281 $\rightarrow$ CM5284 \\
& proC104 str-502 & \\
& (pVS1) & \\
CM5286 & fla-169::Tn5 & CM5281 $\rightarrow$ CM5284 \\
& proC104 str-502 & \\
(pVS1) & \\
CM5287 & fla-169::Tn5 (pVS1) & SC1759 $\rightarrow$ CM5256 \\
SC451 & proC104 & (Barrett et al. 1982) \\
SC1028 & fla-188::Tn5 str-152 & (Ohta et al. 1984) \\
SC1759 & fla-169::Tn5 & SC1045 (Ohta et al. 1984) \\
& proA103 str-140 & \\
& (pVS1) & \\
& &
\end{tabular}

a CM5000 is strain CB15 carried for several years in other laboratories. Growth media and UV irradiation protocols are described elsewhere (Bender 1984)

b $\mathrm{A} \rightarrow \mathrm{B}$ : Conjugal transfer from $\mathrm{A}$ to $\mathrm{B}$ mediated by pVS1

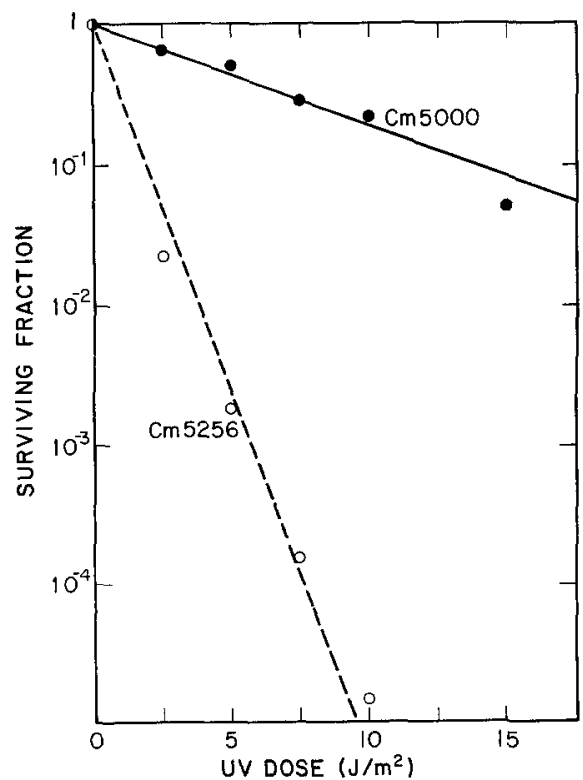

Fig. 1. UV sensitivity of $\mathrm{rec}^{+}$and $\mathrm{rec}-526$ strains. Cultures of strains CM5000 and CM5256 were irradiated as described (Bender 1984). Samples were withdrawn after the indicated doses and the viable count, determined as colony-forming units on broth plates, compared to the viable count before irradiation $\left(3.5 \times 10^{8} / \mathrm{ml}\right.$ for strain CM5000 and $7.4 \times 10^{7} / \mathrm{ml}$ for strain CM5256). $\bullet-$ CM5000; $-\mathrm{O}, \mathrm{CM} 5256$ (rec-526)
Therefore homologous recombinants among the $\mathrm{Km}^{\mathrm{r}}$ transductants will be, in addition to $\mathrm{Km}^{\mathrm{r}}$, non-motile and somewhat filamentous, other $\mathrm{Km}^{\mathrm{R}}$ transductants are presumed to arise from transposition of the $\mathrm{Tn} .5$ element from the donor transducing fragment onto the recipient chromosome.

When a transducing lysate grown on SC1028 was used to transduce either the wild type strain or any of the UV mutant strains listed in Table 1 except CM5256, $\mathrm{Km}^{\mathbf{R}}$ transductants arose at equal frequencies (ca. $10^{-7}-10^{-6}$ per cell) and all the $\mathrm{Km}^{\mathbf{R}}$ transductants were non-motile indicating homologous recombination at the fla locus rather than transposition of $\mathrm{Tn} 5$. However, no $\mathrm{Km}^{\mathrm{R}}$ transductants were observed ( $<0.1 \%$ the wild-type frequency) when the same lysate was used to transduce CM5256. These data suggest that either a) CM5256 is a recombination deficient $\left(\mathrm{Rec}^{-}\right)$ mutant or b) CM5256 is resistant to the transducing phage $\emptyset \mathrm{Cr} 30$. Since we had already shown that $\emptyset \mathrm{Cr} 30$ has an efficiency of plating of 1 on CM5256, we assumed that CM5256 was $\mathrm{Rec}^{-}$and designated the mutation in this strain rec-526.

Strain CM5256 is defective in recombination: RP4 mediated conjugation. It is formally possible that the reduced frequency of transduction observed for CM5256 was the result of some unexpected relationship between the gene mutated and some mechanism necessary for successful transduction. We therefore measured the ability of CM5256 to acquire a fla:: $\operatorname{Tn} 5$ allele by conjugation.

Strain SC1759 [fla-169::Tn5 proA103 str104(pVS1)], like strain $\mathrm{SC1028}$, carries a fla::Tn5 allele (Table 1). This allele allows the distinction between $\mathrm{Km}^{\mathrm{r}}$ transconjugants that are homologous recombinants (non-motile) from those that resulted from transposition of the Tn5 element (motile). Strain SC1759 also carries the proA103 allele allowing counterselection and the streptomycin-resistance $\left(\mathrm{Sm}^{\mathrm{r}}\right)$ marker str-140 which was used here as an unselected donor marker. In addition, strain SC1759 carries the plasmid pVS1 (Barrett et al. 1982), a $\mathrm{Km}^{\mathrm{s}}$ derivative of RP4, as the fertility agent promoting RP4-mediated conjugation and chromosome mobilization.

When strain CM5256 was used as recipient in a mating with strain $\mathrm{SC} 1759, \mathrm{Km}^{\mathrm{r}}$ transconjugants were found at a frequency of only $1 \%$ of that seen when the wild type strain CM5000 was used as recipient (Table 2). As expected, the vast majority (122 of 129 tested) of the $\mathrm{Km}^{\mathrm{r}}$ transconjugants seen when the wild type strain was used as recipient were homologous recombinants (i.e. non-motile). In contrast, a significant portion (28 of 60 tested) of the $\mathrm{Km}^{\mathrm{r}}$ transconjugants seen when the mutant strain CM5256 was used as recipient were motile, suggesting transposition of the Tn 5 element rather than homologous recombination at the fla locus. Furthermore, all of these motile, $\mathrm{Km}^{\mathrm{T}}$ strains (twenty of twenty tested) cotransferred both $\mathrm{Km}^{\mathrm{r}}$ and the tetracycline resistance determinant carried on pVS1 at equally high frequency to $E$. coli during a test cross (data not shown). Thus the $\mathrm{Tn} 5$ element responsible for the $\mathrm{Km}^{\mathrm{r}}$ phenotype of the motile $\mathrm{Km}^{\mathrm{r}}$ transconjugants was presumably located on the pVS1 plasmid.

The remaining $32 \mathrm{Km}^{r}$ transconjugants from the mating with CM5256 as recipient were non-motile and presumably resulted from homologous recombination. These recombinants were then analyzed for the retention of the rec-526 allele by testing for the UV $\mathrm{UV}^{\mathrm{s}}$ phenotype associated with rec526. Thirty-one of these thirty-two recombinants were $\mathrm{UV}^{\mathrm{r}}$, 
Table 2. Conjugal Transfer of fla-169:: Tn5 to CM5256

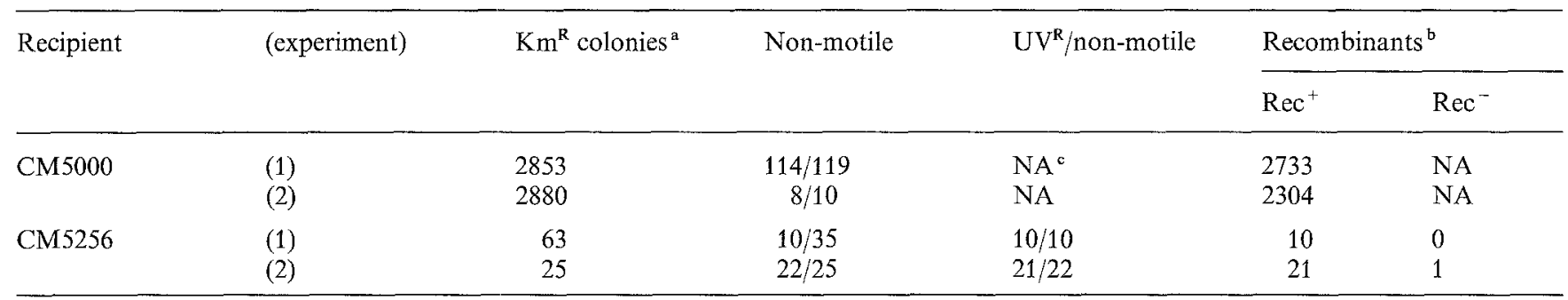

a Filter matings (Ely 1979) with $2 \times 10^{9}$ each of donor (SC1759: fla-169::Tn5, proA103, str-140/pVS1) and recipient were plated on minimal medium containing $\mathrm{Km}$ and lacking proline. Total $\mathrm{Km}^{\mathrm{R}}$ calculated from appropriate dilutions. Several $\mathrm{Km}{ }^{\mathrm{R}}$ colonies were purified by single colony isolation, grown in broth, and examined for motility with the phase contrast microscope to distinguish the homologous recombinants ( $\mathrm{Mot}^{-}$) from the Tn5 mediated transposition events ( $\mathrm{Mot}^{+}$). The homologous recombinants of CM5256 were then tested for $\mathrm{UV}^{\mathrm{s}}$ to distinguish the presence of the rec-526 $\left(\mathrm{UV}^{\mathrm{s}}\right)$ mutation. The $\mathrm{Km}^{\mathrm{R}}$ selection was complicated by the frequent appearance of $\mathrm{Km}^{\mathrm{R}}$ variants on minimal medium, even when the antibiotic concentration was raised to $5 \mathrm{mg} / \mathrm{ml}$. This difficulty was overcome by daily monitoring of the selective plates. Colonies resulting from transfer of the $\mathrm{Tn} 5$-linked $\mathrm{Km}{ }^{\mathrm{R}}$ were visible after 3-5 days whereas colonies resulting from mutation to $\mathrm{Km}^{\mathrm{R}}$ on this medium were very small until 5-6 days

b The recombinants are those $\mathrm{Km}^{\mathrm{R}}$ clones arising from homologous recombination (i.e. non-motile) rather than transposition (i.e. motile). The number is calculated from the total number of $\mathrm{Km}^{\mathrm{R}}$ exconjugants and the fraction of non-motile clones among the total. Those recombinants which are $\mathrm{UV}^{\mathbf{R}}$ are presumed to have arisen in a recombination proficient cytoplasm $\left(\mathrm{Rec}^{+}\right)$; those which are $U^{s}$ may have originated in a recombination deficient cytoplasm ( $\operatorname{Rec}^{-}$), but see text for caveat

- NA, not applicable

suggesting either cotransfer of the $\mathrm{rec}^{+}$allele with fla::Tn5 or reversion of rec-526. (We have not detected reversion of the $\mathrm{UV}^{\mathrm{s}}$ phenotype of rec-526 strains in over one thousand colonies tested.) Only one recombinant (CM5281) appeared in a $\mathrm{UV}^{\mathrm{s}}\left(\mathrm{rec}^{-}\right)$background, in contrast to the more than 5000 recombinants seen in a rec ${ }^{+}$(CM5000) background. Therefore, the recombination frequency seen in strain CM5256 was at most $0.02 \%$ of that seen in the wild type background.

The recombination frequency may actually be lower than $0.02 \%$ of wild type since the incorporation of fla: $: \operatorname{Tn} 5$ may have occurred in a cytoplasm that was transiently $\operatorname{Rec}^{+}$. This possibility arises because the gene defined by rec-526 may be as much as $10 \%$ linked to fla169:: Tn 5 in pVS1 mediated crosses, as suggested above. If $\mathrm{rec}^{+}$were carried into CM5256 along with fla-169:: Tn5, transient expression from the unintegrated fragment might have converted CM5256 into a temporary $\mathrm{Rec}^{+}$cell, allowing incorporation of fla:: $\operatorname{Tn} 5$ with or without the linked $\mathrm{rec}^{+}$allele. Alternatively, the unique recombinant seen in the CM5256 background may represent a low-level residual recombination, particularly in light of the observation that the rec-526 mutation does not totally eliminate Weigle reactivation in C. crescentus (Bender 1984). The remaining formal possibility, that the Tn 5 did not recombine but rather transposed into one of the 26 known fla loci (Johnson and Ely 1979), is unlikely. Most fla mutants are morphologically indistinguishable from wild type. flaO mutants tend to filament (O'Neill and Bender, unpublished) and fla-169 has been mapped to a site in or near flaO (B. Ely and A. Newton, personal communication). The recombinant showed a filamentation pattern identical to the fla-169:: Tn 5 parent and is thus very likely to contain the fla-169:: $\operatorname{Tn} 5$ mutation of the parental (donor) strain.

Thus two lines of evidence suggest that strain CM5256 is recombination deficient $\left(\mathrm{Rec}^{-}\right)$: (1) No recombinants were obtained from $\emptyset \mathrm{Cr} 30$-mediated generalized transduction even though strain CM5256 is fully sensitive to the transducing phage. (2) Only one recombinant was obtained from conjugal transfer experiments under conditions that gave over 5000 recombinants in a wild type background.

The question of whether rec-526 identifies a gene analogous to $\operatorname{rec} A$ of $E$. coli remains open. In support of the analogy, rec-526 causes CM5256 to be very sensitive to UV irradiation. The only recombination gene of $E$. coli whose mutation causes extreme UV sensitivity is $\operatorname{rec} A$ (Clark 1973). Although differences in experimental conditions make comparisons difficult, we found that UV doses resulting in $40 \%$ survival of $\operatorname{Rec}^{+} C$. crescentus resulted in 0.001 to $0.01 \%$ survival of strain CM5256. This result might be compared with the original observation of Clark and Margulies (1965) that a UV dose resulting in $40 \%$ survival of $\operatorname{Rec}^{+} E$. coli resulted in $0.003 \%$ survival of the $\mathrm{Rec}^{-}$strains. Furthermore, the only gene of $E$. coli where single mutations result in at least a $10^{-4}$ reduction in recombination is recA (Clark 1973). Finally the rec-526 mutation reduces the ability of $C$. crescentus to show UV-induced Weigle reactivation of phage $\emptyset \mathrm{CbK}$ (Bender 1984); in $E$. coli, the only recombination deficient mutations that eliminate UV induction of $\mathrm{W}$ reactivation are rec $A$ mutations (Witkin 1976). On the other hand, a slight residuum of Weigle reactivation remains in CM5256 and one recombinant was observed at a frequency of $10^{-4}$ that of wild type. Thus rec-526 may represent either a $r e c A$ analog with a slight residual activity or an allele of a new rec gene different from those seen in E. coli; alternatively, recombination and Weigle-reactivation pathways independent of the wild-type function affected by rec-526 may exist in $C$. crescentus. Isolation of more alleles of this locus and further characterization of rec-526 will be required to distinguish these possibilities.

Genetic transfer of rec-526. In the mating of SC1759 (fla$\left.169:: \mathrm{Tn} 5, \mathrm{rec}^{+}\right)$with CM5256 $\left(\mathrm{fla}^{+}, \mathrm{rec}-526\right), 31$ colonies arose from the apparent cotransfer of $\mathrm{rec}^{+}$along with fla$169:: \operatorname{Tn} 5$ (Table 2). This suggested the possibility of weak 
Table 3. Transfer of rec-526 from $\mathrm{Rec}^{+}$and $\mathrm{Rec}^{-}$donors to CM5284

\begin{tabular}{lccl}
\hline $\begin{array}{l}\text { Donor } \\
\text { (genotype) }\end{array}$ & $\begin{array}{l}\mathrm{Km}^{\mathrm{R}} \text { trans- } \\
\text { conjugants } / \\
2 \times 10^{9} \text { donors }\end{array}$ & $\begin{array}{l}\text { Non- } \\
\text { motile }\end{array}$ & $\begin{array}{l}\mathrm{UV}^{\mathrm{s}} / \\
\text { non-mo- } \\
\text { tile }\end{array}$ \\
\hline $\begin{array}{l}\text { CM5287 } \\
\text { fla-169::Tn5) }\end{array}$ & 2425 & $13 / 18$ & $\mathrm{NA}^{\mathrm{a}}$ \\
$\begin{array}{l}\text { CM5281 } \\
\text { (rec-526, fla-169::Tn5) }\end{array}$ & 55 & $42 / 47$ & $3 / 42$ \\
\hline
\end{tabular}

Filter matings with $2 \times 10^{9}$ each of donor and recipient (CM5284 (proC104, str-502)) were plated on PYE plates supplemented with both $\mathrm{Km}$ and $\mathrm{Sm}$. Total $\mathrm{Km}^{\mathrm{R}}$ recombinants were calculated from appropriate dilutions. Several $\mathrm{Km}^{\mathbf{R}}$ colonies were tested for motility to distinguish homologous recombinants $\left(\mathrm{Mot}^{-}\right)$from illegitimate recombination by transposon $\mathrm{Tn} 5\left(\mathrm{Mot}^{+}\right)$. The $\mathrm{Mot}^{-}$recombinants were then tested for coinheritance of rec-526 by scoring $\mathrm{UV}^{\mathrm{s}}$

The use of streptomycin as a counterselection against $C$. crescentus was very effective unless the strain carried a Tn5 element, in which case $\mathrm{Sm}^{\mathrm{R}}$ variants appeared at higher than the frequency expected from spontaneous mutation to $\mathrm{Sm}^{\mathrm{R}}$ (ca. $10^{-8}$ to $10^{-9}$ in C. crescentus). The Tn 5 element encodes resistance to $20 \mu \mathrm{g} / \mathrm{ml}$ of $\mathrm{Sm}$ in C. crescentus (O'Neill et al. 1984), so high levels of Sm were used $(200 \mu \mathrm{g} / \mathrm{ml})$, the plates were monitored daily, and non-selected markers were used to distinguish $\mathrm{Sm}^{\mathrm{R}}$ variants arising after the matings

a NA, not applicable

linkage between the rec and fla genes. To confirm this linkage, strain CM5281 (fla-169::Tn5, rec-526) was mated with strain CM5284 (proC104, str-502) and the cotransfer of rec-526 with fla:: $\operatorname{Tn} 5$, was tested directly (Table 3 ). Of $47 \mathrm{Km}^{\mathrm{r}}$ transconjugants tested, 42 were non-motile, indicating that these were recombinant for the fla locus. The linkage of rec and fla was confirmed in that three of these 42 recombinants were $U^{s}$ and thus had transferred the rec-526 allele along with fla::Tn 5 . The recombination deficiency of these UV recombinants was confirmed by demonstrating failure of $\emptyset \mathrm{Cr} 30$ mediated transduction to generate $\mathrm{Pro}^{+}$recombinants in one of these strains (not shown).

The data in Table 3 show also that the $\mathrm{Rec}^{-}$strain CM5281 as a donor, generated about 40-fold fewer recombinants in RP4-mediated conjugations than did its isogenic $\mathrm{Rec}^{+}$sibling. The basis for this difference is unknown. It is tempting to speculate that the C. crescentus DNA fragment cloned in the pVS1 plasmid assists in chromosome mobilization by homologous recombination, but Barrett et al. (1982) were unable to detect a difference between RP4mediated and pVS1-mediated transfer. In any event the data in Table 3 show that homologous recombination is not necessary for chromosome mobilization by RP4-like plasmids, since the $\mathrm{Rec}^{-}$strain can serve as a donor, or that if recombination is required, the rec-526 mutation does not entirely eliminate this recombination.

Acknowledgements. We thank B. Ely for generously providing mutant strains and $\mathrm{R}$. Tolsma for excellent technical assistance. This work was supported by grants from the Public Health Service, National Institutes of Health (GM27111 and AI15822) to R.A.B.

\section{References}

Barrett JT, Croft RH, Ferber DM, Gerardot CJ, Schoenlein PV, Ely B (1982) Genetic mapping with Tn5-derived auxotrophs of Caulobacter crescentus. J Bacteriol 151:888-898

Bender RA (1984) Ultraviolet mutagenesis and inducible DNA repair in Caulobacter crescentus. Mol Gen Genet 197:399-402

Bender RA, Agabian N, Shapiro L (1980) Cell differentiation in Caulobacter crescentus. In T Leighton and WF Loomis (ed) The molecular genetics of development, Academic Press New York

Better M, Helinski DR (1983) Isolation and characterization of the rec $A$ gene of Rhizobium meliloti. I Bacteriol 155:311-316

Clark AJ (1973) Recombination deficient mutants of Escherichia coli and other bacteria. Ann Rev Genet 7:67-85

Clark AJ, Margulies AD (1965) Isolation and characterization of recombination-deficient mutants of Escherichia coli K12. Proc Natl Acad Sci USA 53:451-459

Ely B (1979) Transfer of drug resistance factors to the dimorphic bacterium Caulobacter crescentus. Genetics 91:371-380

Johnson RC, Ely B (1979) Analysis of nonmotile mutants of the dimorphic bacterium Caulobacter crescentus. J Bacteriol $137: 627-634$

Little JW, Mount DW (1982) The SOS regulatory system of Escherichia coli. Cell 29:11-22

Milhausen M, Gill PR, Parker G, Agabian N (1982) Cloning of developmentally regulated flagellin genes from Caulobacter crescentus via immunoprecipitation of polyribosomes. Proc Natl Acad Sci USA 79:6847-6851

Ohta N, Swanson E, Ely B, Newton A (1984) Physical mapping and complementation analysis of transposon Tn 5 mutations in Caulobacter crescentus: Organization of transcriptional units in the hook gene cluster. J Bacteriol 158:897-904

O'Neill EA, Kiely GM, Bender RA (1984) Transposon Tn5 encodes streptomycin resistance in nonenteric bacteria. J Bacteriol 159:388-389

Poindexter J (1964) Biological properties and classification of the Caulobacter group. Bacteriol Rev 28:231-295

Purucker M, Bryan R, Amemiya K, Ely B, Shapiro L (1982) Isolation of a Caulobacter gene cluster specifying flagellum production by using nonmotile Tn 5 insertion mutants. Proc Natl Acad Sci USA 79:6797-6801

Witkin EM (1976) Ultraviolet mutagenesis and inducible DNA repair in Escherichia coli. Bacteriol Rev 40:869-907

Communicated by G.R. Smith

Received October 5, 1983 / October 4, 1984 